Canad. Math. Bull. Vol. 21 (1), 1978

\title{
CRITICAL GRAPHS FOR ACYCLIC COLORINGS
}

\author{
BY
}

\author{
DAVID M. BERMAN
}

Introduction. The concept of acyclic colorings of graphs, introduced by Grünbaum [2], is a generalization of point-arboricity. An acyclic coloring of a graph is a proper coloring of its points such that there is no two-colored cycle. We denote by $a(G)$, the acyclic chromatic number of a graph $G$, the minimum number of colors for an acyclic coloring of $G$. We call $G k$-critical if $a(G)=k$ but $a\left(G^{\prime}\right)<k$ for any proper subgraph $G^{\prime}$. For all notation and terminology not defined here, see Harary [3].

Kronk and Mitchem [4] and Bollobás and Harary [1] showed the existence of graphs of every possible order critical for point-arboricity. In this paper we prove the analogous result for acyclic colorings:

THEOREM. For each $k \geq 3$ and $n \geq k$ there exists a $k$-critical graph of order $n$.

We note that the only 2 -critical graph is $K_{2}$.

Proof of the Theorem. We first note without proof the following simple lemma.

LEMMA. If $G=A+B$ then in any acyclic coloring of $G$, either all the points of $A$ or else all the points of $B$ must receive distinct colors.

The theorem is proved by presenting constructions for five classes of critical graphs.

Proposition 1. The theorem is true for $n=2 k-l$, where $5 \leq l \leq k$ and $k$ and $l$ are of the same parity.

Proof. Let $G=\left(\bar{K}_{k-l} \cup K_{l-3}\right)+C_{k-l+3}$. $G$ can be colored either with $k-3$ colors for $\bar{K}_{k-l} \cup K_{l-3}$ and three more for the cycle, or else with $l-3$ colors for $\bar{K}_{k-l} \cup K_{l-3}$ and $k-l+3$ more for the cycle. Thus $a(G)=k$.

To show that $G$ is critical, let $V\left(\bar{K}_{k-l}\right)=\left\{p_{1} \cdots p_{k-l}\right\}, V\left(K_{l-3}\right)=\left\{q_{1} \cdots q_{l-3}\right\}$ and $V\left(C_{k-l+3}\right)=\left\{r_{1} \cdots r_{k-l+3}\right\}$.

(i) Delete line $q_{i}, q_{j}$. Then make $q_{i} ; q_{j}$ and all of $\bar{K}_{k-l}$ color 1 . Use $k-2$ more colors for the remaining $k-2$ points.

Received by the editors March 31, 1977 and revised May 31, 1977. 
(ii) Delete line $r_{i}, r_{i+1}$. Then two-color the cycle and use $k-3$ more colors for $\bar{K}_{k-l} \cup K_{l-3}$.

(iii) Delete line $q_{i}, r_{j}$. Then color the cycle with $k-l+3$ colors. Use the same color for $q_{i}$ as for $r_{j}$ and use $l-4$ more colors for the remaining points of $\bar{K}_{k-l} \cup K_{l-3}$.

(iv) Delete line $p_{i}, r_{j}$. Then make $p_{i}$ and $r_{j}$ both color 1 . Alternate colors 2 and 3 for the rest of the cycle. (Note that since $k-l+3$ is odd the two neighbours of $r_{j}$ are colored differently.) Then use $k-4$ more colors for the remaining $k-4$ points of $\bar{K}_{k-l} \cup K_{l-3}$.

The proofs of Propositions 2 through 5 are similar to that of Proposition 1, and the details are left to the reader.

Proposition 2. The theorem is true for $n=2 k-l$ where $5 \leq l \leq k$, and $k$ and $l$ are of opposite parity.

Proof. Let $G$ be as above, but for each $i$ delete the line $p_{i}, r_{1}$.

PROPOSITION 3. The theorem is true for $n \geq 2 k-4$ where $k$ and $n$ are of the same parity.

Proof. Let $G=\bar{K}_{k-3}+C_{n-k+3}$.

Proposition 4. The theorem is true for $n>2 k-4$ where $k$ and $n$ are of opposite parity.

Proof. Let $G=\bar{K}_{k-3}+C_{n-k+3}$, but for each $i=1$ to $k-3$ delete the line $p_{i}, r_{1}$ for $p_{i} \in \bar{K}_{k-3}$ and $r_{1} \in C_{n-k+3}$.

Proposition 5. The theorem is true for $n=2 k-4$ where $k$ and $n$ are of opposite parity.

Proof. Let $G=\bar{K}_{k-3}+C_{n-k+3}$, but for each $i=2$ to $k-3$ delete the line $p_{i}, r_{1}$ for $p_{i} \in \bar{K}_{k-3}$ and $r_{1} \in C_{n-k+3}$.

\section{REFERENCES}

1. B. Bollobás and F. Harary, Point arboricity critical graphs exist, J. London Math. Soc. (2), 12 (1975), 97-102.

2. B. Grünbaum, "Acyclic colorings of planar graphs", Israel. J. Math., 14 (1973), 390-408.

3. F. Harary, Graph Theory (Addison-Wesley, Reading, Mass., 1969).

4. H. V. Kronk and J. Mitchem, Critical point-arboritic graphs, J. London Math. Soc. (2), 9 (1975), 459-466.

Department of Mathematics,

UNIVERSITY OF NeW ORLEANS,

New Orleans, Louisiana 70122 USA 